\title{
THE DUAL POISSON-LAGUERRE TRANSFORM
}

\author{
BY \\ FRANK M. CHOLEWINSKI AND DEBORAH TEPPER HAIMO( $\left.{ }^{1}\right)$
}

1. Introduction. In a series of papers [1]-[4], [7]-[9], the authors have developed inversion and representation theories for integral transforms whose kernels are functions associated with the fundamental solutions of various generalized heat equations. The present goal is to carry out such a study for the dual PoissonLaguerre transform.

2. Definitions. For $\alpha>-1$, let $L_{n}^{\alpha}(x)$ denote the Laguerre polynomial of degree $n$ given by

$$
L_{n}^{\alpha}(x)=\frac{x^{-\alpha} e^{x}}{n !}\left[\frac{d}{d x}\right]^{n}\left(x^{n+\alpha} e^{-x}\right), \quad n=0,1,2, \ldots
$$

We then have the basic orthogonality relation

$$
\int_{0}^{\infty} L_{n}^{\alpha}(x) L_{m}^{\alpha}(x) d \Omega(x)=\frac{\delta(n, m)}{\rho(n)},
$$

where

$$
\begin{aligned}
d \Omega(x) & =e^{-x} x^{\alpha} d x, \\
\rho(n) & =n ! / \Gamma(n+\alpha+1) .
\end{aligned}
$$

We define the Laguerre differential operator $\nabla_{x}$ by

$$
\begin{aligned}
\nabla_{x} f(x) & =x f^{\prime \prime}(x)+(\alpha+1-x) f^{\prime}(x) \\
& =x^{-\alpha} e^{x} \frac{d}{d x}\left[x^{\alpha+1} e^{-x} \frac{d}{d x} f(x)\right],
\end{aligned}
$$

and note that for the Laguerre polynomial, we have

$$
\nabla_{x} L_{n}^{\alpha}(x)=-n L_{n}^{\alpha}(x), \quad n=0,1, \ldots
$$

Let $f(x)$ be a measurable function defined on $[0, \infty)$. Then its dual Laguerre transform $f^{\sim}(n)$ is given by

$$
f^{\sim}(n)=\int_{0}^{\infty} f(x) \mathscr{L}_{n}^{\alpha}(x) d \Lambda(x)
$$

where

$$
\begin{aligned}
\mathscr{L}_{n}^{\alpha}(x) & =\rho(n) \Gamma(\alpha+1) L_{n}^{\alpha}(x) \\
& ={ }_{1} F_{1}(-n ; 1+\alpha ; x)
\end{aligned}
$$

Received by the editors November 8, 1968.

(1) The research of the first author supported in part by the National Science Foundation, GP-7167, and that of the second author by the National Aeronautics and Space Administration NGR14-008009. 
and

$$
d \Lambda(x)=\frac{1}{\Gamma(\alpha+1)} d \Omega(x)
$$

By inversion, we have

$$
f(x)=\sum_{n=0}^{\infty} f^{\sim}(n) \mathscr{L}_{n}^{\alpha}(x) \sigma(n)
$$

where

$$
\sigma(n)=\frac{1}{\Gamma(\alpha+1) \rho(n)} .
$$

In order to obtain the basic kernels associated with our theory, we derive them heuristically. We need to introduce associated functions and to this end we define formally

$$
d(x, y, z)=\sum_{n=0}^{\infty} \mathscr{L}_{n}^{\alpha}(x) \mathscr{L}_{n}^{\alpha}(y) \mathscr{L}_{n}^{\alpha}(z) \sigma(n)
$$

whence formally

$$
d(x, y, \cdot)^{\sim}(n)=\int_{0}^{\infty} d(x, y, z) \mathscr{L}_{n}^{\alpha}(z) d \Lambda(z)=\mathscr{L}_{n}^{\alpha}(x) \mathscr{L}_{n}^{\alpha}(y) .
$$

We note that $d(x, y, z)$, defined by (2.12), is to be interpreted as the Laguerre analogue of the translates of the Dirac delta function on the real line.

On setting $n=0$ in (2.13), we find that

$$
\int_{0}^{\infty} d(x, y, z) d \Lambda(z)=1 .
$$

We now define the associated function $f(x, y)$ of a function $f(x)$ given for $[0, \infty)$, by

$$
f(x, y)=\int_{0}^{\infty} f(z) d(x, y, z) d \Lambda(z)
$$

whenever the integral converges.

The Laguerre differential heat equation is given by

$$
\nabla_{x} u(x, t)=\partial u(x, t) / \partial t
$$

It is clear that if

$$
u(x, t)=e^{t \nabla_{x}} f(x)
$$

Then, formally,

$$
\partial u(x, t) / \partial t=\nabla_{x} e^{t \nabla_{x}} f(x)=\nabla_{x} u(x, t) .
$$

Taking $f(x)=d(x, 0,0)$, we seek the fundamental solution $g_{\alpha}(x ; t)$ of $(2.16)$ given by

$$
g_{\alpha}(x ; t)=e^{t \nabla_{x}} d(x, 0)
$$


But

$$
\begin{aligned}
e^{t \nabla_{x}} d(x, 0,0) & =e^{t \nabla_{x}} \sum_{n=0}^{\infty} \mathscr{L}_{n}^{\alpha}(x) \sigma(n) \\
& =\sum_{n=0}^{\infty} e^{-n t} \mathscr{L}_{n}^{\alpha}(x) \sigma(n) \\
& =\sum_{n=0}^{\infty} e^{-n t} L_{n}^{\alpha}(x)
\end{aligned}
$$

where we have noted that $e^{t \nabla_{x} \mathscr{L}_{n}^{\alpha}}(x)=e^{-n t} \mathscr{L}_{n}^{\alpha}(x)$. Now by $[5, \mathrm{p} .189(17)]$, we have

$$
g_{\alpha}(x ; t)=\left(\frac{e^{t}}{e^{t}-1}\right)^{\alpha-1} \cdot \exp \left[-x /\left(e^{t}-1\right)\right], \quad t>0 .
$$

Further, we have

$$
\begin{aligned}
g_{\alpha}(x, y ; t) & =\int_{0}^{\infty} d(x, y, z) g_{\alpha}(z ; t) d \Lambda(z) \\
& =\sum_{n=0}^{\infty} \mathscr{L}_{n}^{\alpha}(x) \mathscr{L}_{n}^{\alpha}(y) \sigma(n) \int_{0}^{\infty} \mathscr{L}_{n}^{\alpha}(z) g_{\alpha}(z ; t) d \Lambda(z)
\end{aligned}
$$

But

$$
g_{\alpha}(\cdot ; t)^{\sim}(n)=\int_{0}^{\infty} \mathscr{L}_{n}^{\alpha}(z) g_{\alpha}(z ; t) d \Lambda(z)=e^{-n t}
$$

and so

$$
\begin{aligned}
g_{\alpha}(x, y ; t) & =\sum_{n=0}^{\infty} e^{-n t} \mathscr{L}_{n}^{\alpha}(x) \mathscr{L}_{n}^{\alpha}(y) \sigma(n) \\
& =\left(\frac{e^{t}}{e^{t}-1}\right)^{\alpha+1} \exp \left[-(x+y) /\left(e^{t}-1\right)\right] \mathscr{F}\left(\frac{2\left(x y e^{t}\right)^{1 / 2}}{e^{t}-1}\right)
\end{aligned}
$$

by $[5$, p. $189(19)]$, where

$$
\mathscr{I}(z)=2^{\alpha} \Gamma(\alpha+1) z^{-\alpha} I_{\alpha}(z),
$$

$I_{\alpha}(z)$ being the Bessel function of imaginary argument. Henceforth, we shall write $g(x, y ; t)$ for $g_{\alpha}(x, y ; t)$, including the subscript only for functions of order $\neq \alpha$. We also have

$$
g(x, \cdot ; t) \sim(n)=e^{-n t} \mathscr{L}_{n}^{\alpha}(x) .
$$

The function $g(x, y ; t)$ is the kernel of our transformation. From its explicit form (2.22), it is immediate that

$$
g(x, y ; t)>0, \quad t>0 .
$$

Further, it is well known that $g(x, y ; t)$ is a variation diminishing kernel; see [13]. 
For a function $\phi$ defined for $[0, \infty)$, the dual Poisson-Laguerre transform is given by

$$
u(x, t)=\int_{0}^{\infty} g(x, y ; t) \phi(y) d \Lambda(y), \quad t>0,
$$

whenever the integral converges, whereas the dual Poisson-Laguerre-Stieltjes transform for a function $\alpha$ of bounded variation is defined by

$$
u(x, t)=\int_{0}^{\infty} g(x, y ; t) d \alpha(y), \quad t>0 .
$$

Examples of functions which are dual Poisson-Laguerre transforms are given in the following table:

$$
u(x, t)
$$

$\phi(y)$

1. 1

2. $e^{-t}\left[x+(1+\alpha)\left(e^{t}-1\right)\right] \quad y$

3. $n !\left(\frac{e^{t}-1}{e^{t}}\right)^{n} L_{n}^{\alpha}\left(-\frac{x}{e^{t}-1}\right) \quad y^{n}$

4. $e^{-\operatorname{tn}} \mathscr{L}_{n}^{\alpha}(x)$

$\mathscr{L}_{n}^{\alpha}(y)$

5. $x^{n}$

$$
n !(-1) !\left(e^{t}-1\right)^{n} L_{n}^{\alpha}\left(\frac{x e^{t}}{e^{t}-1}\right) .
$$

3. Properties of the kernel $g(x, y ; t)$. The function $g(x, y, t)$ plays a central role in our theory and in this section we study some of its fundamental properties. By (2.24), we have

$$
\int_{0}^{\infty} g(x, y ; t) \mathscr{L}_{n}^{\alpha}(y) d \Lambda(y)=e^{-n t} \mathscr{L}_{n}^{\alpha}(x) .
$$

On setting, in turn, $n=0,1,2$ in (3.1),we readily establish the following result.

THEOREM 3.1. For $x, t$ fixed, $t>0$,

$$
\begin{aligned}
\int_{0}^{\infty} g(x, y ; t) d \Lambda(y)= & 1, \\
\int_{0}^{\infty} y g(x, y ; t) d \Lambda(y)= & (\alpha+1)\left(1-e^{-t}\right)+x e^{-t}, \\
\int_{0}^{\infty} y^{2} g(x, y ; t) d \Lambda(y)= & (\alpha+2)(\alpha+1)\left(1-e^{-t}\right)^{2} \\
& +2(\alpha+2)\left(1-e^{-t}\right) e^{-t} x+e^{-2 t} x^{2} .
\end{aligned}
$$

We next state a useful property which follows readily from the fact that, for $x>0$,

$$
\begin{aligned}
g(x, y ; t) \sim & \frac{\Gamma(\alpha+1)}{2\left(\pi\left(e^{t}-1\right)\right)^{1 / 2}} e^{t[(\alpha / 2)+(3 / 4)]}(x y)^{-(\alpha / 2)-(1 / 4)} e^{x} \\
& \times \exp \left[-\frac{\left(\left(x e^{t}\right)^{1 / 2}-y^{1 / 2}\right)^{2}}{e^{t}-1}\right], \quad y \rightarrow \infty
\end{aligned}
$$


THEOREM 3.2. For $x$ fixed, $\delta$ any positive number, and $0 \leqq y \leqq x-\delta, x+\delta \leqq y<\infty$,

$$
\lim _{t \rightarrow 0^{+}} g(x, y ; t)=0 .
$$

This has an important consequence.

THEOREM 3.3. For $x$ fixed,

$$
\begin{aligned}
\lim _{t \rightarrow 0^{+}} \int_{a}^{b} g(x, y ; t) d \Lambda(y) & =0, & & 0 \leqq a \leqq b<x<\infty \\
& =0, & & 0 \leqq x<a \leqq b \leqq \infty \\
& =1, & & 0 \leqq a<x<b \leqq \infty
\end{aligned}
$$

Proof. (3.7) and (3.8) follow from the preceding theorem and an appeal to Lebesgue's dominated convergence theorem which holds since, in (3.5),

$$
\frac{\exp \left[t\left(\frac{\alpha}{2}+\frac{3}{4}\right)-\frac{\left(\left(x e^{t}\right)^{1 / 2}-y^{1 / 2}\right)^{2}}{e^{t}-1}\right]}{\left(e^{t}-1\right)^{1 / 2}}
$$

is uniformly bounded in any finite interval of the positive $t$-axis, and the dominant integral $\int_{a}^{b} y^{-\alpha / 2-1 / 4} d \Lambda(y)<\infty$. (3.9) is a direct result of the first two cases and (3.2).

The following basic relation of Huygens type may be established by an appeal to the definition of $g(x, y ; t)$ and an evaluation of the integral [6, p. 52 (23)].

THEOREM 3.4. For $t_{1}, t_{2}>0$,

$$
\int_{0}^{\infty} g\left(x, z ; t_{1}\right) g\left(y, z ; t_{2}\right) d \Lambda(z)=g\left(x, y ; t_{1}+t_{2}\right)
$$

For $x, x_{0}$ fixed, the quotient

$$
\mathscr{G}(y)=\frac{g(x, y ; t)}{g\left(x_{0}, y ; 1\right)}, \quad 0<t<1,
$$

plays a significant role in the development of the theory and we now explore, in particular, its changes of trend. To this end we need some preliminary lemmas.

LeMma 3.5. For $x, x_{0}$ fixed, $0 \leqq x, x_{0}<\infty, 0<t<1$,

$$
\lim _{y \rightarrow \infty} \frac{g(x, y ; t)}{g\left(x_{0}, y ; 1\right)}=0 .
$$

Proof. The result follows immediately by (3.5).

LEMma 3.6. If $\Delta(s)=1-|s|$ for $|s|<1$ and $\Delta s=0$ otherwise, then

$$
g(x, y ; t)=\lim _{h \rightarrow 0} \int_{0}^{\infty} g(u, y ; t) \frac{\Gamma(\alpha+1) \Delta((x-u) / h)}{h u^{\alpha} e^{-u}} d \Lambda(u) .
$$


Proof. We have

$$
\begin{aligned}
I & =\int_{0}^{\infty} g(u, y ; t) \frac{\Gamma(\alpha+1) \Delta((x-u) / h)}{h u^{\alpha} e^{-u}} d \Lambda(u) \\
& =\int_{x-h}^{x+h} g(u, y ; t) \frac{\Delta((x-u) / h)}{h} d u .
\end{aligned}
$$

By a change of variables, we have

$$
I=\int_{-1}^{1} g(x-u h, y ; t) \Delta(u) d u,
$$

so that an appeal to Lebesgue's dominated convergence theorem yields

$$
\lim _{h \rightarrow 0} I=g(x, y ; t) \int_{-1}^{1} \Delta(u) d u=g(x, y ; t)
$$

and the lemma is established.

LEMMA 3.7. For any real number $a, x, x_{0}$ fixed, and $0<t<1$,

$$
g\left(x_{0}, y ; 1\right)-a g(x, y ; t)
$$

has at most two variations of sign for $0 \leqq y<\infty$.

Proof. By (3.10) and (3.13), we note that

$$
\begin{aligned}
& g\left(x_{0}, y ; 1\right)-a g(x, y ; t) \\
& =\lim _{h \rightarrow 0} \int_{0}^{\infty} g(u, y ; t)\left[g\left(x_{0}, u ; 1-t\right)-\frac{a \Gamma(\alpha+1) \Delta((x-u) / h)}{h u^{\alpha} e^{-u}}\right] d \Lambda(u) .
\end{aligned}
$$

Since $g(x, y ; t)$ is a variation diminishing kernel, it follows that the number of variations of sign of

$$
\int_{0}^{\infty} g(u, y ; t)\left[g\left(x_{0}, u ; 1-t\right)-\frac{a \Gamma(\alpha+1) \Delta((x-u) / h)}{h u^{\alpha} e^{-u}}\right] d \Lambda(u)
$$

does not exceed that of

$$
\left[g\left(x_{0}, y ; 1-t\right)-\frac{a \Gamma(\alpha+1) \Delta((x-y) / h)}{h y^{\alpha} e^{-y}}\right]
$$

and so by $[12$, p. 81$]$, the number of variations of sign of $g\left(x_{0}, y ; 1\right)-a g(x, y ; t)$ does not exceed that of

$$
\liminf _{h \rightarrow 0^{+}}\left[g\left(x_{0}, y ; 1-t\right)-\frac{a \Gamma(\alpha+1) \Delta((x-y) / h)}{h y^{\alpha} e^{-y}}\right] .
$$

Since for $x, x_{0}$ fixed and $h$ sufficiently small, (3.15) has at most two changes of sign, so does (3.14) and the proof is complete.

We now investigate the changes of trend of $\mathscr{G}(y)$.

THEOREM 3.8. $\mathscr{G}(y)$ defined as in (3.11) has at most one change of trend. 
Proof. Let $a$ be a real number and consider

$$
g\left(x_{0}, y ; 1\right)-a g(x, y ; t)=a g\left(x_{0}, y ; 1\right)[1 / a-\mathscr{G}(y)] .
$$

Clearly the number of variations of sign of the function on the left is equal to that of $1 / a-\mathscr{G}(y)$. If $\mathscr{G}(y)$ were to have more than two changes of trend, there would necessarily exist a real number $a$ for which the number of variations of sign of $g\left(x_{0}, y ; 1\right)-a g(x, y ; t)$ would exceed 2 , contradicting the previous lemma. On the other hand, if $\mathscr{G}(y)$ were to have two changes of trend, since $\mathscr{G}(y)>0$, then either $\mathscr{G}(y) \uparrow$ in the neighborhood of $y=0$, and hence would have to decrease once and then increase for all large $y$, contradicting (3.12) so that in this case $\mathscr{G}(y)$ must have exactly one change of trend; or else, $\mathscr{G}(y) \downarrow$ in the neighborhood of $y=0$, and since (3.12) holds, the assumption that $\mathscr{G}(y)$ has two changes of trend would imply the existence of a real number $a$ such that the number of variations of sign of $g\left(x_{0}, y ; 1\right)$ $-\operatorname{ag}(x, y ; t)$ is 3 , contradicting Lemma 3.7 , so that in this case $\mathscr{G}(y)$ has no change in trend and the result is established.

We now strengthen this theorem.

THEOREM 3.9. For $x, x_{0}$ fixed, $\delta$ any positive number and $t$ sufficiently small, $g(x, y ; t) / g\left(x_{0}, y ; 1\right)$ has exactly one change of trend in $(x-\delta, x+\delta)$.

Proof. Because of the preceding theorem, it is enough to prove that the quotient has at least one change in trend. To this end, if $A$ is a real number, $0<A<1$, then there exists a $y$ in the interval $(x-\delta, x-\delta / 2)$ such that $\mathscr{G}(y)<A$. For otherwise, we have $\mathscr{G}(y) \geqq 1$ for all $y, x-\delta<y<x-\delta / 2$. But then, by (3.7), we have

$$
\begin{aligned}
0 & =\lim _{t \rightarrow 0^{+}} \int_{x-\delta}^{x-\delta / 2} g(x, y ; t) d \Lambda(y) \\
& =\lim _{t \rightarrow 0^{+}} \int_{x-\delta}^{x-\delta / 2} \mathscr{G}(y) g\left(x_{0}, y ; 1\right) d \Lambda(y) \\
& \geqq A \int_{x-\delta}^{x-\delta / 2} g\left(x_{0}, y ; 1\right) d \Lambda(y) \geqq A>0,
\end{aligned}
$$

a contradiction. Similarly we may see that in the interval $(x+\delta / 2, x+\delta)$, there exists a $y$ for which $\mathscr{G}(y)<A$. Finally in the interval $(x-\delta / 2, x+\delta / 2)$, there exists a $y$ for which $\mathscr{G}(y)>A$; for, otherwise, $\mathscr{G}(y) \leqq 0$ for all $y$ in that interval and we would have, by (3.9)

$$
1=\lim _{t \rightarrow 0^{+}} \int_{x-\delta / 2}^{x+\delta / 2} g(x, y ; t) d \Lambda(y) \leqq A<1,
$$

a contradiction. We have thus proved the theorem.

Similar results hold for the changes of trend of the kernel $g(x, y ; t)$ itself. In order to prove these, we need a preliminary lemma.

LEMMA 3.10. If

$$
g_{\varepsilon}(x, y ; t)=\int_{0}^{\infty} g(x, u ; 1 / \varepsilon) g(u, y ; t) d \Lambda(u), \quad \varepsilon>0,
$$


then

$$
\lim _{\varepsilon \rightarrow 0^{+}} \frac{g_{\varepsilon}(x, y ; t)}{g(x, x ; 1 / \varepsilon)}=1 .
$$

Proof. Let $\delta>0$. Then for $x>\delta$ we have, by (2.22) and the standard asymptotic estimate for $\mathscr{I}(z),[5$, p. 86],

and

$$
\frac{g(x, y ; 1 / \varepsilon)}{g(x, x ; 1 / \varepsilon)} \leqq K \frac{e^{x}}{\delta^{\alpha / 2+1 / 4}} \quad \text { for } \delta \leqq y
$$

Also, clearly,

$$
\frac{g(x, y ; 1 / \varepsilon)}{g(x, x ; 1 / \varepsilon)} \leqq K \quad \text { for } 0 \leqq y \leqq \delta
$$

$$
\lim _{\varepsilon \rightarrow 0^{+}} \frac{g(x, y ; 1 / \varepsilon)}{g(x, x ; 1 / \varepsilon)}=1
$$

Hence, applying Lebesgue's dominated convergence theorem to

we have

$$
\frac{g_{\varepsilon}(x, y ; t)}{g(x, x ; 1 / \varepsilon)}=\int_{0}^{\infty} \frac{g(x, u ; 1 / \varepsilon)}{g(x, x ; 1 / \varepsilon)} g(u, y ; t) d \Lambda(u),
$$

$$
\lim _{\varepsilon \rightarrow 0^{+}} \frac{g_{\varepsilon}(x, y ; t)}{g(x, x ; 1 / \varepsilon)}=\int_{0}^{\infty} g(u, y ; t) d \Lambda(u)=1
$$

and the lemma is proved.

Corresponding to Lemma 3.7, we have the following result.

LEMMA 3.11. For any real number $a$, and $x$ fixed,

$$
g(x, y ; t)-a
$$

has at most two variations of sign for $0 \leqq y<\infty$.

Proof. By (3.13) and (3.16), we have

$$
\begin{aligned}
g(x, y ; t)- & a g_{\varepsilon}(x, y ; t) \\
& =\lim _{h \rightarrow 0} \int_{0}^{\infty} g(u, y ; t)\left[\frac{\Delta((x-u) / h) \Gamma(\alpha+1)}{h u^{\alpha} e^{-u}}-a g(x, u ; 1 / \varepsilon)\right] d \Lambda(u) .
\end{aligned}
$$

Hence an argument entirely analogous to that of Lemma 3.7, establishes that $g(x, y ; t)-a g_{\varepsilon}(x, y ; t)$ has at most two variations of sign. Since $a$ is arbitrary, set $a=a^{*} / g(x, x ; 1 / \varepsilon)$ with $a^{*}$ any real number. Then the number of variations of sign of

$$
g(x, y ; t)-a^{*} g_{\varepsilon}(x, y ; t) / g(x, x ; 1 / \varepsilon)
$$

does not exceed 2. By [12, p. 84], it follows that the number of variations of sign of $g(x, y ; t)-a^{*}$ is bounded by lim $\sup _{\varepsilon \rightarrow 0}$ of the number of variations of sign of

$$
g(x, y ; t)-a^{*} g_{\varepsilon}(x, y ; t) / g(x, x ; 1 / \varepsilon)
$$

and the proof is complete. 
By an argument entirely analogous to that used to establish Theorem 3.9, we have the following parallel result.

THEOREM 3.12. For $x$ fixed, and $\delta>0, g(x, y ; t)$ has at least one variation of trend in $(x-\delta, x+\delta)$ for $t$ sufficiently small.

This theorem together with a proof of Theorem 3.8 in which the function $g\left(x_{0}, y ; 1\right)$ has been replaced by 1 , enables us to reach the following conclusion.

THEOREM 3.13. For $x$ fixed, $\delta>0, g(x, y ; t)$ has exactly one change of trend in $(x-\delta, x+\delta)$ for $t$ sufficiently small.

From the definition (2.22) and elementary estimates we also have, for $x$ fixed, $0<t \leqq 1$

$$
\lim _{y \rightarrow \infty} g(x, y ; t)=0 .
$$

4. Estimates of functions of $g(x, y ; t)$. In order to derive estimates of the kernels, of quotients of kernels, and of derivations of these, we need the following useful identities.

$$
\begin{aligned}
& \frac{\partial}{\partial x} g(x, y ; t)=-\frac{1}{e^{t}-1} g(x, y ; t)+\frac{y}{e^{t}-1} g_{\alpha+1}(x, y ; t) . \\
& \nabla_{x} g(x, y ; t)=\left[\frac{(x+y) e^{t}}{\left(e^{t}-1\right)^{2}}-\frac{\alpha+1}{e^{t}-1}\right] g(x, y ; t)-\frac{x y}{e^{t}-1} g_{\alpha+1}(x, y ; t) . \\
& \frac{\partial}{\partial y} \frac{g(x, y ; t)}{g\left(x_{0}, y ; t_{0}\right)}=-\frac{e^{t_{0}}-e^{t}}{\left(e^{t_{0}}-1\right)\left(e^{t}-1\right)} \frac{g(x, y ; t)}{g\left(x_{0}, y ; t_{0}\right)}+\frac{x}{e^{t}-1} \frac{g_{\alpha+1}(x, y ; t)}{g\left(x_{0}, y ; t_{0}\right)} \\
& -\frac{x_{0}}{e^{t_{0}}-1} \frac{g(x, y ; t) g_{\alpha+1}\left(x_{0}, y ; t_{0}\right)}{\left[g\left(x_{0}, y ; t_{0}\right)\right]^{2}} \text {. } \\
& \frac{\partial}{\partial y} \frac{\nabla_{x} g(x, y ; t)}{g\left(x_{0}, y ; t_{0}\right)}=\left[\frac{e^{t}}{\left(e^{t}-1\right)^{2}}-\frac{y e^{t}}{\left(e^{t}-1\right)^{3}}\right] \frac{g(x, y ; t)}{g\left(x_{0}, y ; t_{0}\right)} \\
& +\left[\frac{x y\left(2 e^{t}-1\right)}{\left(e^{t}-1\right)^{3}}-\frac{x}{e^{t}-1}\right] \frac{g_{\alpha+1}(x, y ; t)}{g\left(x_{0}, y ; t_{0}\right)} \\
& -\frac{x^{2} y}{\left(e^{t}-1\right)^{2}} \frac{g_{\alpha+2}(x, y ; t)}{g\left(x_{0}, y ; t\right)}+\frac{1}{e^{t_{0}}-1} \frac{\nabla_{x} g(x, y ; t)}{g\left(x_{0}, y ; t_{0}\right)} \\
& -\frac{x_{0}}{e^{t_{0}}-1} \frac{\left[\nabla_{x} g(x, y ; t)\right] g_{\alpha+1}\left(x_{0}, y ; t_{0}\right)}{\left[g\left(x_{0}, y ; t_{0}\right)\right]^{2}} \text {. }
\end{aligned}
$$

By appealing to the definitions and standard asymptotic expansions, we may establish the following theorem. 
THEOREM 4.1. For $x, x_{0}$ fixed, $t_{0}>t>0$,

$$
\begin{aligned}
& g(x, y ; t) \sim \Gamma(\alpha+1) \frac{\left(e^{t}\right)^{(\alpha / 2)+(3 / 4)}}{2\left(\pi\left(e^{t}-1\right)\right)^{1 / 2}} e^{x}(x y)^{-(\alpha / 2)-(1 / 4)} \exp \left[-\frac{\left(x^{1 / 2} e^{t / 2}-y^{1 / 2}\right)^{2}}{e^{t}-1}\right], \\
& y \rightarrow \infty
\end{aligned}
$$

$$
\frac{g(x, y ; t)}{g\left(x_{0}, y ; t_{0}\right)} \sim\left(e^{t-t_{0}}\right)^{(\alpha / 2)+(3 / 4)}\left(\frac{\left(e^{t_{0}}-1\right)}{\left(e^{t}-1\right)}\right)^{1 / 2}\left(\frac{x_{0}}{x}\right)^{(\alpha / 2)+(1 / 4)} \exp \left[\frac{\left(x^{1 / 2} e^{t_{0} / 2}-x_{0}^{1 / 2} e^{t / 2}\right)^{2}}{e^{t_{0}}-e^{t}}\right]
$$

$$
\times \exp \left\{-\frac{e^{t_{0}}-e^{t}}{\left(e^{t}-1\right)\left(e^{t_{0}}-1\right)}\left[y^{1 / 2}-\frac{x^{1 / 2} e^{t / 2}\left(e^{t_{0}}-1\right)-x_{0}^{1 / 2} e^{t_{0} / 2}\left(e^{t}-1\right)}{\iota^{t_{0}}-e^{t}}\right]^{2}\right\}
$$

$$
y \rightarrow \infty
$$

$$
\begin{aligned}
\frac{\partial}{\partial y} \frac{g(x, y ; t)}{g\left(x_{0}, y ; t\right)} \sim\left[\frac{e^{t}-e^{t_{0}}}{\left(e^{t}-1\right)\left(e^{t_{0}}-1\right)}+\frac{x}{e^{t}-1}\left(x y e^{-t}\right)^{-1 / 2}\right. & \left.-\frac{x_{0}}{e^{t_{0}}-1}\left(x_{0} y e^{-t_{0}}\right)^{-1 / 2}\right] \\
& \times \frac{g(x, y ; t)}{g\left(x_{0}, y ; t_{0}\right)}, \quad y \rightarrow \infty
\end{aligned}
$$

The elementary inequality

$$
-\frac{\left(\left(x e^{t}\right)^{1 / 2}-y^{1 / 2}\right)^{2}}{e^{t}-1} \leqq \frac{A x e^{t}}{1-A\left(e^{t}-1\right)}-A y, \quad t>0, \quad A<1 /\left(e^{t}-1\right)
$$

enables us to derive the following estimate.

TheOREM 4.2. For $x$ fixed, $t>0, \delta>0$,

$$
g(x, y ; t)=O\left(\exp \left[-\frac{y}{e^{t+\delta}-1}\right]\right), \quad y \rightarrow \infty .
$$

5. Convergence. We next study the convergence behavior of the dual PoissonLaguerre-Stieltjes transform.

THEOREM 5.1. If $\alpha$ is a function of bounded variation in every finite interval, and if

$$
\int_{0}^{\infty} g\left(x_{0}, y ; c\right) d \alpha(y)=A
$$

for some $x_{0} \geqq 0, c>0$, then $\int_{0}^{\infty} g(x, y ; t) d \alpha(y), 0 \leqq x<\infty, 0<t \leqq c$ converges and

$$
\lim _{t \rightarrow c^{-}} \int_{0}^{\infty} g\left(x_{0}, y ; t\right) d \alpha(y)=A \text {. }
$$

Proof. We have

$$
I=\int_{0}^{\infty} g(x, y ; t) d \alpha(y)=\int_{0}^{\infty} \frac{g(x, y ; t)}{g\left(x_{0}, y ; c\right)} d \beta(y),
$$

where

$$
\beta(y)=\int_{0}^{y} g\left(x_{0}, u ; c\right) d \alpha(u), \quad 0 \leqq y<\infty
$$


Now $\beta(y)$ is of bounded variation in every finite interval, $\beta(\infty)$ exists by hypothesis, and $g(x, y ; t) / g\left(x_{0}, y ; c\right)=\mathscr{G}(y)$ is a positive, continuous function which eventually is nonincreasing. It follows by [12, p. 174] that the integral $I$ converges for $0 \leqq x<\infty$, $0<t \leqq c$. Further, since $\int_{0}^{\infty} g\left(x_{0}, y ; t\right) d \alpha(y)$ is a continuous function of $t$ in $0<t \leqq c$, its limit as $t \rightarrow c^{-}$is $A$.

This enables us to prove the following stronger result.

THEOREM 5.2. If the integral

$$
\int_{0}^{\infty} g(s, y ; t) d \alpha(y), \quad s=\sigma+i \tau,
$$

converges for $s=\sigma_{0} \geqq 0$, then the integral converges locally uniformly in the complex plane, and represents there an analytic function.

Proof. Let $S$ be any compact set in the complex plane. For $R>0$, Let $S \subseteq$ $\{s|| s \mid \leqq R\}$. Then if $s=\sigma+i \tau \in S$, we have, by (2.22) and the estimate $|\mathscr{I}(z)|$ $\leqq \mathscr{I}(|z|)$,

$$
|g(s, y ; t)| \leqq \exp \left[2 R /\left(e^{t}-1\right)\right] g(R, y ; t)
$$

It follows that

$$
\left|\int_{0}^{\infty} g(s, y ; t) d \alpha(y)\right| \leqq \exp \left[2 R /\left(e^{t}-1\right)\right] \int_{0}^{\infty} g(R, y ; t) d \alpha(y) .
$$

By the preceding theorem, the convergence of the integral (5.3) for $s=\sigma_{0}$ implies its convergence for $0 \leqq \sigma<\alpha$ and hence the integral on the right side of inequality (5.5) converges. An appeal to the Weierstrass $M$-test completes the proof.

6. Inversion. We now turn to a fundamental inversion theorem for the dual Poisson-Laguerre transform under general conditions.

THEOREM 6.1. Let $\phi$ be integrable in every finite interval and let

$$
\int_{0}^{\infty} g\left(x_{0}, y ; 1\right) \phi(y) d \Lambda(y)
$$

converge for some $x_{0} \geqq 0$. If

$$
\lim _{h \rightarrow 0^{+}} \frac{1}{h} \int_{x}^{x+h}[\phi(y)-\phi(x)] d \Lambda(y)=0,
$$

then

$$
\lim _{t \rightarrow 0^{+}} \int_{0}^{\infty} g(x, y ; t) \phi(y) d \Lambda(y)=\phi(x)
$$

Proof. Set

$$
\psi(y)=\int_{x}^{y}[\phi(u)-\phi(x)] d \Lambda(u)
$$


Then, by hypothesis, it follows that for a given $\varepsilon>0$, there exists a $\delta>0$ such that

$$
|\psi(y)|<\varepsilon|y-x| \quad \text { if }|y-x|<\delta .
$$

With $x$ and $\delta$ fixed, let

$$
\begin{aligned}
I & =\int_{0}^{\infty} g(x, y ; t) \phi(y) d \Lambda(y)=\left(\int_{0}^{x-\delta}+\int_{x-\delta}^{x+\delta}+\int_{x+\delta}^{\infty}\right) g(x, y ; t) \phi(y) d \Lambda(y) \\
& =I_{1}+I_{2}+I_{3} .
\end{aligned}
$$

Now

where

$$
\begin{aligned}
I_{3} & =\int_{x+\delta}^{\infty} \frac{g(x, y ; t)}{g\left(x_{0}, y ; 1\right)} g\left(x_{0}, y ; 1\right) \phi(y) d \Lambda(y) \\
& =\left.\frac{g(x, y ; t)}{g\left(x_{0}, y ; 1\right)} A(y)\right|_{x+\delta} ^{\infty}-\int_{x+\delta}^{\infty} A(y) d\left[\frac{g(x, y ; t)}{g\left(x_{0}, y ; 1\right)}\right],
\end{aligned}
$$

$$
A(y)=\int_{x+\delta}^{y} g\left(x_{0}, y ; 1\right) \phi(y) d \Lambda(y) .
$$

Since $A(\infty)$ exists, $\mathscr{G}(y)=g(x, y ; t) / g\left(x_{0}, y ; 1\right)$ tends to zero for $0<t<1$ as $y \rightarrow \infty$, and $A(x+\delta)=0$, the integrated part vanishes, and we have

$$
I_{3}=-\int_{x+\delta}^{\infty} A(y) d \mathscr{G}(y) .
$$

By, Theorems 3.2 and 3.9 , for $t$ sufficiently small and $y \geqq x+\delta, \mathscr{G}(y) \downarrow$. Hence

$$
\begin{aligned}
\left|I_{3}\right| & \leqq M \int_{x+\delta}^{\infty} d\left[-\frac{g(x, y ; t)}{g\left(x_{0}, y ; 1\right)}\right] \\
& =M \frac{g(x, x+\delta ; t)}{g\left(x_{0}, x+\delta ; 1\right)}=o(1) \quad \text { as } t \rightarrow 0^{+}
\end{aligned}
$$

Further, we have

$$
I_{1}=\int_{0}^{x-\delta} \frac{g(x, y ; t)}{g\left(x_{0}, y ; 1\right)} g\left(x_{0}, y ; 1\right) \phi(y) d \Lambda(y)=\int_{0}^{x-\delta} \frac{g(x, y ; t)}{g\left(x_{0}, y ; 1\right)} d B(y)
$$

where

$$
B(y)=\int_{0}^{y} g\left(x_{0}, y ; 1\right) \phi(y) d \Lambda(y) .
$$

Since $B(0)=0$, we have

$$
I_{1}=\frac{g(x, x-\delta ; t)}{g\left(x_{0}, x-\delta ; 1\right)} B(x-\delta)-\int_{0}^{x-\delta} B(y) d\left[\frac{g(x, y ; t)}{g\left(x_{0}, y ; 1\right)}\right]
$$

so that

$$
\begin{aligned}
\left|I_{1}\right| & \leqq M \frac{g(x, x-\delta ; t)}{g\left(x_{0}, x-\delta ; 1\right)}+M \int_{0}^{x-\delta} d\left[\frac{g(x, y ; t)}{g\left(x_{0}, y ; 1\right)}\right] \\
& \leqq 2 M \frac{g(x, x-\delta ; t)}{g\left(x_{0}, x-\delta ; 1\right)}-\frac{g(x ; t)}{g\left(x_{0} ; 1\right)}=o(1) \quad \text { as } t \rightarrow 0^{+}
\end{aligned}
$$


Finally, consider

$$
\begin{aligned}
J= & \int_{x-\delta}^{x+\delta} g(x, y ; t)[\phi(y)-\phi(x)] d \Lambda(y)=\int_{x-\delta}^{x+\delta} g(x, y ; t) d \psi(y) \\
= & g(x, x+\delta ; t) \psi(x+\delta)-g(x, x-\delta ; t) \psi(x-\delta)-\int_{x-\delta}^{x+\delta} \psi(y) d[g(x, y ; t)] \\
= & g(x, x+\delta ; t) \psi(x+\delta)-g(x, x-\delta ; t) \psi(x-\delta) \\
& -\frac{1}{e^{t}-1} \int_{x-\delta}^{x+\delta} \psi(y)\left[x g_{\alpha+1}(x, y ; t)-g(x, y ; t)\right] d y
\end{aligned}
$$

by (4.1). Hence

$$
|J| \leqq o(1)+\frac{\varepsilon}{e^{t}-1} \int_{x-\delta}^{x+\delta}|y-x|\left|x g_{\alpha+1}(x, y ; t)-g(x, y ; t)\right| d y, \quad t \rightarrow 0^{+}
$$

and since the integral is finite,

From the fact that

$$
|J| \leqq o(1)+\varepsilon, \quad t \rightarrow 0^{+} .
$$

it follows that

$$
\limsup _{t \rightarrow 0^{+}} \int_{x-\delta}^{x+\delta} g(x, y ; t) d \Lambda(y)=1
$$

$$
\limsup _{t \rightarrow 0^{+}}|J|=\limsup _{t \rightarrow 0^{+}}\left|I_{2}-\phi(x)\right| \leqq \varepsilon
$$

Thus

$$
\limsup _{t \rightarrow 0^{+}}|I-\phi(x)| \leqq \varepsilon
$$

and the proof is complete.

Next we establish the order of magnitude of a function whose dual PoissonLaguerre-Stieltjes transform converges.

THEOREM 6.2. Let $\alpha(y)$ be of bounded variation in every finite interval of $0<y<\infty$. If, for $x, t$ fixed, $0 \leqq x<\infty, 0<t<1$,

$$
\int_{0}^{\infty} g(x, y ; t) d \alpha(y)
$$

converges, then

$$
\alpha(y)=o\left(\frac{1}{g(x, y ; t)}\right), \quad y \rightarrow \infty,
$$

and $\alpha\left(0^{+}\right)$exists.

Proof. As a consequence of Theorem 3.12 and by (3.18), we may choose numbers $y_{1}, y_{2}$ so that $x<y_{1}<y_{2}$ and so large that $g(x, y ; t) \downarrow$ for $y_{1} \leqq y \leqq y_{2}$. Now

$$
\begin{aligned}
\alpha\left(y_{2}\right)-\alpha\left(y_{1}\right) & =\int_{y_{1}}^{y_{2}} d \alpha(y)=\int_{y_{1}}^{y_{2}} \frac{g(x, y ; t)}{g(x, y ; t)} d \alpha(y) \\
& =\frac{1}{g\left(x, y_{1} ; t\right)} \int_{y_{1}}^{\eta} g(x, y ; t) d \alpha(y),
\end{aligned}
$$


where the last equation follows from the mean value theorem with $y_{1} \leqq \eta \leqq y_{2}$. Since the integral on the right converges, for given $\varepsilon>0$, there exists a number $y_{0}>0$ so that

$$
\alpha\left(y_{2}\right)-\alpha\left(y_{1}\right)<\frac{\varepsilon}{g\left(x, y_{1} ; t\right)}, \quad y_{1}>y_{0},
$$

or, since $g\left(x, y_{1} ; t\right)>0$,

$$
\left[\alpha\left(y_{2}\right)-\alpha\left(y_{1}\right)\right] g\left(x, y_{1} ; t\right)<\varepsilon .
$$

Further, $g\left(x, y_{2} ; t\right)<g\left(x, y_{1} ; t\right)$ since $g(x, y ; t) \downarrow$ for $y_{1} \leqq y \leqq y_{2}$, and so

$$
\left[\alpha\left(y_{2}\right)-\alpha\left(y_{1}\right)\right] g\left(x, y_{2} ; t\right)<\varepsilon .
$$

Hence

$$
\begin{aligned}
\left|\alpha\left(y_{2}\right)\right| g\left(x, y_{2} ; t\right) & \leqq\left|\alpha\left(y_{2}\right)-\alpha\left(y_{1}\right)\right| g\left(x, y_{2} ; t\right)+\left|\alpha\left(y_{1}\right)\right| g\left(x, y_{2} ; t\right) \\
& <\varepsilon+\left|\alpha\left(y_{1}\right)\right| g\left(x, y_{2} ; t\right) .
\end{aligned}
$$

Since $\left|\alpha\left(y_{1}\right)\right| g\left(x, y_{2} ; t\right) \rightarrow 0$ as $y_{2} \rightarrow \infty$, by (3.18), we have (6.5). To verify that $\alpha\left(0^{+}\right)$exists, we choose numbers $y_{1}, y_{2}$ such that $0<y_{1}<y_{2}<x$ and so small that $g(x, y ; t) \uparrow$ for $y_{1} \leqq y \leqq y_{2}$. Then by an appeal to the mean value theorem,

$$
\alpha\left(y_{2}\right)-\alpha\left(y_{1}\right)=\frac{1}{g\left(x, y_{2} ; t\right)} \int_{\eta}^{y_{2}} g(x, y ; t) d \alpha(y),
$$

where $y_{1} \leqq \eta \leqq y_{2}$. From this it follows that as $y_{2} \rightarrow 0$,

$$
\alpha\left(y_{2}\right)-\alpha\left(y_{1}\right)=o(1)
$$

and hence $\alpha\left(0^{+}\right)$exists.

7. Asymptotic estimates of dual Poisson-Laguerre transforms. We now study the growth patterns of convergent dual Poisson-Laguerre transforms. To this end, we need the following elementary inequalities.

LEMMA 7.1. For $B>0$,

$$
\int_{0}^{\infty} \exp \left[-B\left(y^{1 / 2}-A\right)^{2}\right] d y \leqq \frac{2 A}{B^{1 / 2}} \pi^{1 / 2}
$$

and

$$
\int_{0}^{\infty} \frac{1}{y^{1 / 2}} \exp \left[-B\left(y^{1 / 2}-A\right)^{2}\right] d y \leqq \frac{2 \pi^{1 / 2}}{B^{1 / 2}} .
$$

Proof. (7.1) is established by a simple change of variable. We have

$$
\begin{aligned}
\int_{0}^{\infty} \exp \left[-B\left(y^{1 / 2}-A\right)^{2}\right] d y & \leqq \int_{-\infty}^{\infty} \exp \left[-B\left(|y|^{1 / 2}-A\right)^{2}\right] d y \\
& =\frac{2}{B^{1 / 2}} \int_{-\infty}^{\infty} e^{-\theta^{2}}\left(\frac{\theta}{B^{1 / 2}}+A\right) d \theta \\
& =2 A \pi^{1 / 2} / B^{1 / 2}
\end{aligned}
$$


and (7.2) similarly,

$$
\int_{0}^{\infty} \frac{\exp \left[-B\left(y^{1 / 2}-A\right)^{2}\right] d y}{y^{1 / 2}}=2 \int_{-A}^{\infty} e^{-B \theta^{2}} d \theta \leqq 2 \int_{-\infty}^{\infty} e^{-B \theta^{2}} d \theta=2 \pi^{1 / 2} / B^{1 / 2} .
$$

We now develop our first asymptotic estimate.

THEOREM 7.2. Let

$$
u(x, t)=\int_{0}^{\infty} g(x, y ; t) d \alpha(y)
$$

converge for $\left(x_{0}, t_{0}\right), 0 \leqq x_{0}<\infty, t_{0}>0$. Then for $t$ fixed, $0<t<t_{0}$,

$$
u(x, t)=O\left(x^{(1 / 4)-(\alpha / 2)} \exp \left[x /\left(1-e^{t-t_{0}}\right)\right]\right), \quad x \rightarrow \infty .
$$

Proof. We have

$$
u(x, t)=\int_{0}^{\infty} \frac{g(x, y ; t)}{g\left(x_{0}, y ; t_{0}\right)} d \beta(y)
$$

where

$$
\beta(y)=\int_{0}^{y} g\left(x_{0}, y ; t_{0}\right) d \alpha(y) .
$$

Integrating by parts and noting that the integrated term vanishes, we find that

$$
u(x, t)=-\int_{0}^{\infty} \beta(y) \frac{\partial}{\partial y} \frac{g(x, y ; t)}{g\left(x_{0}, y ; t_{0}\right)} d y .
$$

An appeal to (4.7) and (4.8) yields, since $\beta(y)$ is bounded for $0 \leqq y \leqq \infty$,

$$
\begin{aligned}
|u(x, t)| \leqq & K \frac{\exp \left[x e^{t_{0}} /\left(e^{t_{0}}-e^{t}\right)\right]}{x^{(\alpha / 2)+(1 / 4)}} \int_{0}^{\infty}\left|\frac{e^{t_{0}}-e^{t}}{\left(e^{t}-1\right)\left(e^{t_{0}}-1\right)}+\left(\frac{\left(x e^{t}\right)^{1 / 2}}{e^{t}-1}+\frac{\left(x_{0} e^{t_{0}}\right)^{1 / 2}}{e^{t_{0}}-1}\right) \frac{1}{y^{1 / 2}}\right| \\
& \times \exp \left\{\frac{e^{t_{0}}-e^{t}}{\left(e^{t}-1\right)\left(e^{t_{0}}-1\right)}\left[y^{1 / 2}-\frac{x^{1 / 2} e^{t / 2}\left(e^{t_{0}}-1\right)-x_{0}^{1 / 2} e^{t_{0} / 2}\left(e^{t}-1\right)}{e^{t_{0}}-e^{t}}\right]^{2}\right\} d y,
\end{aligned}
$$

where $K$ is dependent on $t, t_{0}$, and $x_{0}$, but not on $x$. Applying the preceding lemma, we have (7.4), the result sought.

The conclusion of the theorem may be sharpened as in the following corollary.

Corollary 7.3. Let

$$
u(x, t)=\int_{0}^{\infty} g(x, y ; t) d \alpha(y)
$$

converge for $0<t<c, 0 \leqq x<\infty$. Then for any fixed $t$ and for any $t_{0}, 0<t<t_{0}<c$,

$$
u(x, t)=O\left(\exp \left[x /\left(1-e^{t-t_{0}}\right)\right]\right), \quad x \rightarrow \infty .
$$

Proof. Since we have, by the theorem,

$$
u(x, t)=O\left(x^{(1 / 4)-(\alpha / 2)} \exp \left[x /\left(1-e^{t-t_{0}}\right)\right]\right), \quad x \rightarrow \infty,
$$


and this implies for a smaller $t_{0}$,

$$
u(x, t)=O\left(\exp \left[x /\left(1-e^{t-t_{0}}\right)\right]\right), \quad x \rightarrow \infty
$$

the proof is complete since $t_{0}$ is arbitrary.

This may be extended further to yield the following estimate.

Corollary 7.4. Let

$$
u(x, t)=\int_{0}^{\infty} g(x, y ; t) d \alpha(y)
$$

converge for $0<t<c, 0 \leqq x<\infty$. Then, if $0<\delta<c / 2$,

$$
u(x, t)=O\left(\exp \left[x /\left(1-e^{-\delta / 2}\right)\right]\right), \quad x \rightarrow \infty,
$$

uniformly for $\delta \leqq t \leqq c-\delta$.

Proof. By the preceding corollary, taking $t_{0}=c-\delta / 2$, we have

$$
|u(x, t)| \leqq A(t) \exp \left[x /\left(1-e^{t-c+\delta / 2}\right)\right]
$$

or, for $\delta \leqq t \leqq c-\delta$

$$
|u(x, t)| \leqq A \exp \left[x /\left(1-e^{-\delta / 2}\right)\right],
$$

where $A$ is the maximum of $A(t)$ in the interval $\delta \leqq t \leqq c-\delta$, and is finite, as is clear from the inequality of the theorem.

Next we establish the growth behavior of the incomplete Poisson-LaguerreStieltjes transform $\int_{0}^{A} g(x, y ; t) d \alpha(y), A>0$.

THEOREM 7.5. Let

$$
u(x, t)=\int_{0}^{A} g(x, y ; t) d \alpha(y), \quad 0 \leqq x<\infty, \quad A>0,
$$

where $\alpha(y)$ is of bounded variation in every finite interval of $[0, \infty)$. Then, for $0<t<c$,

$$
u(x, t)=O\left(1 / x^{\alpha+1}\right), \quad x \rightarrow \infty .
$$

Proof. We have

$$
g(x, y ; t) \leqq K(x y)^{-(\alpha / 2)-(1 / 4)} e^{y} \exp \left\{-\left[x^{1 / 2}-\left(y e^{t}\right)^{1 / 2}\right]^{2} /\left(e^{t}-1\right)\right\} .
$$

But, clearly,

Hence

$$
\exp \left\{-\left[x^{1 / 2}-\left(y e^{t}\right)^{1 / 2}\right]^{2} /\left(e^{t}-1\right)\right\}<\frac{k}{\left|x^{1 / 2}-\left(y e^{t}\right)^{1 / 2}\right|^{2(\alpha+1)}}
$$

$$
g(x, y ; t)<K \frac{e^{y}(x y)^{-(\alpha / 2)-(1 / 4)}}{\left(x^{1 / 2}-\left(y e^{t}\right)^{1 / 2}\right)^{2(\alpha+1)}} .
$$

In addition, for $\delta>0$, it follows from the definition (2.22) that, for $0 \leqq y \leqq \delta$,

$$
g(x, y ; t) \leqq K \exp \left[\left(2\left(x \delta e^{t}\right)^{1 / 2}-x\right) /\left(e^{t}-1\right)\right] .
$$


Hence

$$
\begin{aligned}
|u(x, t)| \leqq & K \exp \frac{\left[\left(2\left(x \delta e^{t}\right)^{1 / 2}-x\right) /\left(e^{t}-1\right)\right]}{\exp \left[\left(2\left(x \delta e^{t}\right)^{1 / 2}-x\right) /\left(e^{t}-1\right)\right]} \int_{0}^{\delta}|d \alpha(y)| \\
& +\frac{K e^{A} \int_{\delta}^{A}|d \alpha(y)|}{\left|x^{1 / 2}-\left(A e^{c}\right)^{1 / 2}\right|^{2(\alpha+1)}\left(A e^{c} \delta\right)^{(\alpha / 2)+(1 / 4)}}, \quad x>A e^{c} \\
= & O\left(1 / x^{\alpha+1}\right), \quad x \rightarrow \infty
\end{aligned}
$$

and (7.10) is established.

8. Dual Laguerre temperatures. We study now solutions of the Laguerre differential heat equation. We introduce the following notation for the class of such functions.

Definition 8.1. A $C^{2}$ function $u(x, t)$ which satisfies the Laguerre differential heat equation

$$
\nabla_{x} u(x, t)=\partial u(x, t) / \partial t
$$

where $\nabla_{x}$ is defined in (2.5), in a domain $D$, is said to belong to class $H$ there and is called a dual Laguerre temperature. The function $u(x, t) \in H$ in a region $R$ if there exists a domain $D$ with $R \subseteq D$ and $u(x, t) \in H$ in $D$. A convergent dual Poisson-Laguerre-Stieltjes transform is a dual Laguerre temperature as established in the following theorem.

THEOREM 8.2. Let $\alpha$ be of bounded variation in every finite interval and let

$$
u(x, t)=\int_{0}^{\infty} g(x, y ; t) d \alpha(y), \quad 0 \leqq x<\infty,
$$

the integral converging for $0<t<c$. Then $u(x, t) \in H$ there and $u(s, t), s=\sigma+i \tau$, is analytic in any bounded domain.

Proof. The analyticity of $u(x, t)$ follows from Theorem 5.2. Membership in $H$ is a consequence of the fact that

$$
\int_{0}^{\infty} \nabla_{x} g(x, y ; t) d \alpha(y)
$$

converges uniformly for $x$ in any finite interval and $0<t<c$. That this is so may be established by noting that, by hypothesis,

$$
\int_{0}^{\infty} g\left(x, y ; t_{0}\right) d \alpha(y)<\infty, \quad 0 \leqq x<\infty,
$$

for $t_{0}$ such that $0<\delta \leqq t \leqq c-\delta<t_{0}<c$. Now

$$
\begin{aligned}
I & =\int_{T_{1}}^{T_{2}} \nabla_{x} g(x, y ; t) d \alpha(y)=\int_{T_{1}}^{T_{2}} \frac{\nabla_{x} g(x, y ; t)}{g\left(x, y ; t_{0}\right)} g\left(x, y ; t_{0}\right) d \alpha(y) \\
& \left.=-\frac{\nabla_{x} g(x, y ; t)}{g\left(x, y ; t_{0}\right)} \beta(y)\right]_{T_{1}}^{T_{2}}+\int_{T_{1}}^{T_{2}} \beta(y) \frac{\partial}{\partial y}\left[\frac{\nabla_{x} g(x, y ; t)}{g\left(x, y ; t_{0}\right)}\right] d y,
\end{aligned}
$$


where

$$
\beta(y)=\int_{y}^{\infty} g\left(x, y ; t_{0}\right) d y=o(1), \quad y \rightarrow \infty .
$$

Since by (4.2), (4.4), (4.5), (4.6), we have for $0 \leqq x \leqq R, R$ any positive number and for $0<t<c$,

$$
\begin{gathered}
\frac{\nabla_{x} g(x, y ; t)}{g\left(x, y ; t_{0}\right)}=O(1), \quad y \rightarrow \infty \\
\frac{\partial}{\partial y} \frac{\nabla_{x} g(x, y ; t)}{g\left(x, y ; t_{0}\right)}=O\left(e^{-A y}\right), \quad y \rightarrow \infty, A \text { a positive constant, }
\end{gathered}
$$

uniformly, $\lim _{T_{1}, T_{2} \rightarrow \infty} I=0$ uniformly and the proof is complete.

We next prove that a dual Laguerre temperature, in a strip $0<t \leqq c$, which vanishes along $t=0^{+}$, vanishes identically throughout the strip. To this end, we need a preliminary result.

THEOREM 8.3. Let $u(x, t) \in H$ for $0 \leqq x \leqq R, 0<t \leqq c$, and let

$$
\begin{gathered}
u(R, t) \geqq 0, \quad 0<t \leqq c, \\
\lim _{t \rightarrow 0^{+} ; x \rightarrow x_{0}} u(x, t) \geqq 0 \quad \text { for all } x_{0}, 0 \leqq x_{0} \leqq R .
\end{gathered}
$$

Then

$$
u(x, t) \geqq 0, \quad 0 \leqq x \leqq R, 0<t \leqq c .
$$

Proof. By (8.3), it follows that for $\varepsilon>0$, there is a number $\delta>0$ such that

$$
u(x, t)>-\varepsilon \quad \text { for } 0 \leqq x \leqq R, 0<t \leqq \delta .
$$

Assuming that the conclusion of the theorem is false, there must exist a point $\left(x_{1}, t_{1}\right), 0 \leqq x_{1}<R, 0<t_{1} \leqq c$ for which

$$
u\left(x_{1}, t_{1}\right)=-A<0 .
$$

Let

$$
v(x, t)=u(x, t)+r\left(t-t_{1}\right),
$$

where $r>0$ is such that $r t_{1}<A$, and let $\varepsilon$ be such that $\varepsilon<A-r t_{1}$. Now

$$
v\left(x_{1}, t_{1}\right)=u\left(x_{1}, t_{1}\right)=-A .
$$

Also

$$
\begin{aligned}
v(x, t) & =u(x, t)+r\left(t-t_{1}\right) \\
& >-\varepsilon+r t-r t_{1} \\
& >-\varepsilon-r t_{1} \\
& >-A
\end{aligned}
$$


for $0 \leqq x \leqq R, 0<t \leqq \delta$. Hence $v(x, t)$ must assume a minimum at a point $\left(x_{2}, t_{2}\right)$ with $0 \leqq x_{2} \leqq R, \delta<t_{2} \leqq c$. We then have,

$$
\begin{aligned}
\partial v\left(x_{2}, t_{2}\right) / \partial x=0, & 0<x_{2}<R, \delta<t_{2}<c, \\
\partial^{2} v\left(x_{2}, t_{2}\right) / \partial x^{2} \geqq 0, & 0<x_{2}<R, \delta<t_{2}<c,
\end{aligned}
$$

whereas, if the minimum occurs along $x_{2}=0$, then

$$
\partial v\left(0, t_{2}\right) / \partial x \geqq 0, \quad \delta<t_{2} \leqq c,
$$

and if the minimum occurs along $t_{2}=c$, then

$$
\partial v\left(x_{2}, t_{2}\right) / \partial t \leqq 0, \quad 0 \leqq x_{2} \leqq R .
$$

It thus follows that

$$
\left(\nabla_{x}-\frac{\partial}{\partial t}\right) v\left(x_{2}, t_{2}\right)=x_{2} \frac{\partial^{2}}{\partial x^{2}} v\left(x_{2}, t_{2}\right)+\left(\alpha+1-x_{2}\right) \frac{\partial}{\partial x} v\left(x_{2}, t_{2}\right)-\frac{\partial}{\partial t} v\left(x_{2}, t_{2}\right) \geqq 0 .
$$

On the other hand,

$$
\left(\nabla_{x}-\frac{\partial}{\partial t}\right) v\left(x_{2}, t_{2}\right)=\left(\nabla_{x}-\frac{\partial}{\partial x}\right) u\left(x_{2}, t_{2}\right)-r=-r<0,
$$

since $u \in H$. The contradiction negates the assumption that the conclusion of the theorem is false and (8.4) is established.

We now prove the principal result.

THEOREM 8.4. Let $u(x, t) \in H$ for $0<t \leqq c$, and assume that

$$
\begin{gathered}
\lim _{x \rightarrow x_{0} ; t \rightarrow 0^{+}} u(x, t)=0 \text { for all } x_{0}, 0 \leqq x_{0}<\infty, \\
f(x)=\max _{0<t \leqq c}|u(x, t)|=O\left(e^{a x}\right), \quad x \rightarrow \infty, \text { for some } a .
\end{gathered}
$$

Then

$$
u(x, t)=0, \quad 0<t \leqq c, 0 \leqq x<\infty .
$$

Proof. By the asymptotic estimates for $g(x, x ; t)$, there exists a constant $K$ such that $K g(R, R ; t) \geqq 1$ for $t>0, R \geqq R_{0}$. Let $v_{ \pm}(x, t)=K f(R) g(x, R ; t) \pm u(x, t)$.

Then, clearly, $v_{ \pm}(x, t) \in H$ for $0 \leqq x \leqq R, 0<t \leqq c$. Further,

$$
\begin{aligned}
v_{ \pm}(R, t) & =K f(R) g(R, R ; t) \pm u(R, t) \\
& \geqq f(R) \pm u(R, t) \geqq 0,
\end{aligned}
$$

since $K g(R, R ; t) \geqq 1$ and $f(R)=\max _{0<t \leqq c}|u(R, t)|$. We have, also,

$$
\liminf _{t \rightarrow 0^{+} ; x \rightarrow x_{0}} v_{ \pm}(R, t) \geqq \liminf _{t \rightarrow 0^{+} ; x \rightarrow x_{0}}[ \pm u(x, t)]= \pm \liminf _{t \rightarrow 0^{+} ; x \rightarrow x_{0}} u(x, t)=0,
$$

by hypothesis. The conditions of the preceding theorem are thus satisfied and

$$
v_{ \pm}(x, t) \geqq 0, \quad 0 \leqq x \leqq R, 0<t \leqq c
$$


or, by the definition of $v_{ \pm}(x, t)$,

$$
K f(R) g(x, R ; t) \geqq|u(x, t)| .
$$

Now (8.6) and (4.10) yields

$$
|u(x, t)| \leqq K e^{a R} \exp \left[-R /\left(e^{t+\delta}-1\right)\right]
$$

Holding $x$ and $t$ fixed and letting $R \rightarrow \infty$, we have $u(x, t)=0$ for $t<\ln (1+1 / a)-\delta$, or since $\delta$ may be chosen arbitrarily close to zero, for $t<\ln (1+1 / a)$. The proof is thus complete if $c<\ln (1+1 / a)$. Otherwise the result may be derived by repeating the argument for $u(x, t+\ln (1+1 / a))$ instead of $u(x, t)$.

9. Positive dual Laguerre temperatures. A nonnegative dual Laguerre temperature which vanishes at time zero, vanishes identically. To establish this fact, we need, first, the following result.

THEOREM 9.1. If $u(x, t) \geqq 0$ is in $H$ for $0<t \leqq c, 0 \leqq x<\infty$, then

$$
\int_{0}^{\infty} g(x, y ; t) u(y, \delta) d \Lambda(y)
$$

converges for $0<t<c-\delta, 0<\delta<c$, and

$$
\int_{0}^{\infty} g(x, y ; t) u(y, \delta) d \Lambda(y) \leqq u(x, t+\delta), \quad 0<t<c-\delta .
$$

Proof. Set

$$
v(x, t)=u(x, t+\delta)-\int_{0}^{A} g(x, y ; t) u(y, \delta) d \Lambda(y),
$$

where $A$ and $\delta$ are arbitrary positive numbers with $\delta<c$. We will show that $v(x, t) \geqq 0$ for $0<t<c-\delta$. Clearly $v(x, t) \in H$ there and by the proof of Theorem 6.1, we have

$$
\begin{aligned}
\lim _{x \rightarrow x_{0}: t \rightarrow 0^{+}} v(x, t) & =u\left(x_{0}, \delta\right) \geqq 0, & & x_{0}>A, \\
& =0, & & x_{0}<A .
\end{aligned}
$$

Also

$$
\lim _{x \rightarrow A ; t \rightarrow 0^{+}} \int_{0}^{A} g(x, y ; t) u(y, \delta) d \Lambda(y) \leqq u(A, \delta),
$$

so that

$$
\liminf _{x \rightarrow A ; t \rightarrow 0^{+}} v(x, t) \geqq u(A, \delta)-u(A, \delta)=0
$$

Hence for all $x_{0}$,

$$
\liminf _{x \rightarrow x_{0} ; t \rightarrow 0^{+}} v(x, t) \geqq 0 .
$$

Let us now assume that $v(x, t)$ is not nonnegative for $0<t<c-\delta$ so that

$$
v\left(x_{0}, t_{0}\right)=-a<0
$$


at some point $\left(x_{0}, t_{0}\right), 0<t_{0}<c-\delta$. Since, by $(4.5), \int_{0}^{A} g(x, y ; t) u(y, \delta) d \Lambda(y)$ converges uniformly to zero for $t>0$, as $x \rightarrow \infty$, and since $u(x, t) \geqq 0$, we can choose $R$ so large that

$$
v(R, t) \geqq-a / 2, \quad 0<t<c-\delta .
$$

The function $v(x, t)+a / 2$ satisfies the conditions of Theorem 8.3 and so $v(x, t)$ $\geqq-a / 2$ for $0 \leqq x \leqq \infty, 0<t<c-\delta$, contradicting (9.2). It follows that our assumption must be false and

$$
\int_{0}^{A} g(x, y ; t) u(y, \delta) d \Lambda(y) \leqq u(x, t+\delta)
$$

for $0<t<c-\delta$, and since the integral increases with $A$, the convergence of $\int_{0}^{\infty} g(x, y ; t) u(y, \delta) d \Lambda(y)$ is assured and the theorem is established.

We now have the means to prove the principal result.

TheOREM 9.2. If $u(x, t) \geqq 0$ is in $H$ for $0<t \leqq c$, and if $u(x, 0)=0,0 \leqq x<\infty$, then $u(x, t) \equiv 0$ for $0 \leqq x<\infty, 0<t \leqq c$.

Proof. Let

$$
v(x, t)=\int_{0}^{t} u(x, z) d z, \quad 0<t \leqq c .
$$

Then $v(x, t) \geqq 0,0<t \leqq c$, and $v(x, 0)=0$. Also,

$$
\partial v(x, t) / \partial t=u(x, t)
$$

and

$$
\begin{aligned}
\nabla_{x} v(x, t) & =\int_{0}^{t} \nabla_{x} u(x, z) d z \\
& =\int_{0}^{t} \frac{\partial}{\partial z} u(x, z) d z \\
& =u(x, t)-u\left(x, 0^{+}\right) \\
& =u(x, t)
\end{aligned}
$$

so that $v(x, t) \in H$ for $0 \leqq t \leqq c$. Hence $v(x, t)$ satisfies all the hypotheses of the theorem. Furthermore, $v(x, t) \uparrow$ as a function of $t$. Also, since

$$
\nabla_{x} v(x, t)=x^{-\alpha} e^{x} \frac{\partial}{\partial x}\left[x^{\alpha+1} e^{-x} \frac{\partial}{\partial x} v(x, t)\right]
$$

we have

$$
x^{\alpha+1} e^{-x} \frac{\partial}{\partial x} v(x, t)=\int_{0}^{x} x^{\alpha} e^{-x} u(x, t) d x \geqq 0,
$$

so that $v(x, t) \uparrow$ as a function of $x$. Since $u(x, t) \equiv 0$ if $v(x, t) \equiv 0$, there is no loss of generality in assigning to $u(x, t)$ the additional monotonic properties of $v(x, t)$. 
Now, since $u(x, t) \geqq 0$ and is nondecreasing in $t$, it follows that, for an arbitrary $\delta, 0<\delta<c$,

$$
f(x)=\max _{0 \leqq t \leqq \delta} u(x, t)=u(x, \delta) .
$$

Further, since $f(x)$ is nondecreasing, we have, for $x>0$,

$$
\frac{2^{\alpha+1}-1}{\Gamma(\alpha+2)} f(x) e^{-2 x} x^{\alpha+1} \leqq \int_{x}^{2 x} f(y) d \Lambda(y),
$$

or, for $t_{0}, 0<t_{0}<c-\delta$,

$$
\begin{aligned}
\frac{2^{\alpha+1}-1}{\Gamma(\alpha+2)} f(x) e^{-2 x} x^{\alpha+1} g\left(2 x ; t_{0}\right) & \leqq \int_{x}^{2 x} g\left(y ; t_{0}\right) f(y) d \Lambda(y) \\
& =\int_{x}^{2 x} g\left(y ; t_{0}\right) u(y, \delta) d \Lambda(y) \\
& \leqq \int_{0}^{\infty} g\left(y ; t_{0}\right) u(y, \delta) d \Lambda(y) .
\end{aligned}
$$

By the preceding theorem, with $x=0$, the integral on the right converges. Hence

$$
f(x)=O\left(\exp \left[2 x e^{t_{0}} /\left(e^{t_{0}}-1\right)\right]\right), \quad x \rightarrow \infty .
$$

The hypotheses of Theorem 8.4 thus hold, and it follows that $u(x, t)=0$ in the strip $0<t<\delta$ and since $\delta$ is arbitrary, the proof is complete.

As a consequence of this result we may strengthen the preceding theorem as follows.

Corollary 9.3. If $u(x, t) \geqq 0$ is in $H$ for $0<t \leqq c$, then

$$
u(x, t+\delta)=\int_{0}^{\infty} g(x, y ; t) u(y, \delta) d \Lambda(y), \quad 0<t<c-\delta .
$$

Proof. We have, by Theorem 9.3, that

$$
v(x, t)=u(x, t+\delta)-\int_{0}^{\infty} g(x, y ; t) u(y, \delta) d \Lambda(y) \geqq 0, \quad 0<t<c-\delta .
$$

Then, clearly, $v(x, t) \in H, 0<t \leqq c$, and $v(x, 0)=0$. The hypotheses of Theorem 9.2 thus hold for $v(x, t)$ so that $v(x, t) \equiv 0$ for $0<t<c-\delta$, and the conclusion of the corollary is valid:

10. The Huygens property. We consider next a subclass $H^{*}$ of the class $H$ of dual Laguerre temperatures, consisting of dual Laguerre temperatures $u(x, t)$ for $a<t<b$ for which

$$
u(x, t)=\int_{0}^{\infty} g\left(x, y ; t-t^{\prime}\right) u\left(y, t^{\prime}\right) d \Lambda(y)
$$

the integral converging for every $t, t^{\prime}, a<t^{\prime}<t<b$. Such functions are said to have the Huygens property for $a<t<b$. 
We establish that a function represented by a dual Poisson-Laguerre-Stieltjes integral belongs to the class $H^{*}$ in the region of convergence of the integral. To this end, we need a preliminary theorem.

TheOREM 10.1. Let $u(x, t) \in H,|u(x, t)|<K e^{A x}$ for $a<t<b$. Then

$$
u(x, t)=\int_{0}^{\infty} g\left(x, y ; t-t^{\prime}\right) u\left(y, t^{\prime}\right) d \Lambda(y), \quad a<t^{\prime}<t<b,
$$

the equality holding over that part of $t^{\prime}<t<b$ for which the integral converges absolutely.

Proof. Let

$$
v(x, t)=\int_{0}^{\infty} g\left(x, y ; t-t^{\prime}\right) u\left(y, t^{\prime}\right) d \Lambda(y),
$$

with the integral converging absolutely for $t^{\prime}<t<p \leqq b$. If $\left(x_{0}, t_{0}\right)$ is a point such that $0 \leqq x_{0}<\infty, t^{\prime}<t<t_{0}<p \leqq b$, then

$$
\begin{aligned}
|v(x, t)| & \leqq \int_{0}^{\infty} \frac{g\left(x, y ; t-t^{\prime}\right)}{g\left(x_{0}, y ; t_{0}-t^{\prime}\right)} g\left(x_{0}, y ; t_{0}-t^{\prime}\right)\left|u\left(y, t^{\prime}\right)\right| d \Lambda(y) \\
& \leqq K \int_{0}^{\infty} \exp \left[-\frac{x+y}{e^{t-t^{\prime}}-1}+\frac{x_{0}+y}{e^{t_{0}-t^{\prime}}-1}\right] \exp \left[\frac{2\left(x y e^{t-t^{\prime}}\right)^{1 / 2}}{e^{t-t^{\prime}}-1}\right] \\
\times & \times g\left(x_{0}, y ; t_{0}-t^{\prime}\right)\left|u\left(y, t^{\prime}\right)\right| d \Lambda(y) \\
& \leqq K \int_{0}^{\infty} \exp -\left[\frac{\left(\left(x e^{t-t^{\prime}}\right)^{1 / 2}-y\right)^{2}}{e^{t-t^{\prime}}-1}\right] e^{x} \exp \left[\frac{\left(x_{0}+y\right)}{e^{t_{0}-t^{\prime}}-1}\right] \\
\times & \quad g\left(x_{0}, y ; t_{0}-t^{\prime}\right)\left|u\left(y, t^{\prime}\right)\right| d \Lambda(y),
\end{aligned}
$$

or taking, in inequality (4.12), $A=1 /\left(e^{t_{0}-t^{\prime}}-1\right)$, we have

$$
\begin{aligned}
|v(x, t)| \leqq K \int_{0}^{\infty} \exp \left[\frac{x e^{t-t^{\prime}}}{e^{t_{0}-t^{\prime}}-e^{t-t^{\prime}}-1}-\frac{y}{e^{t_{0}-t^{\prime}}-1}\right] \exp \left[x+\frac{x_{0}+y}{e^{t_{0}-t^{\prime}}-1}\right] \\
\leqq K \exp \left[x \frac{e^{t_{0}-t^{\prime}}-1}{e^{t_{0}-t^{\prime}}-e^{t-t^{\prime}}-1}+\frac{x}{e^{t_{0}-t^{\prime}}-1}\right] \\
\times \int_{0}^{\infty} g\left(x_{0}, y ; t_{0}-t^{\prime}\right)\left|u\left(y, t^{\prime}\right)\right| d \Lambda(y)
\end{aligned}
$$

By the assumed convergence of the integral on the right, it follows that $|v(x, t)|$ $\leqq K e^{A x}$ uniformly for $t^{\prime}<t<p-\delta$. Now, set

$$
w(x, t)=u(x, t)-v(x, t) .
$$

Since $u \in H$ by hypothesis and $v \in H$ by virtue of being a convergent dual PoissonLaguerre transform it follows that $w \in H$. Further $|w| \leqq K e^{A x}$ uniformly for $t^{\prime}<t<p-\delta$. Moreover, by Theorem $6.1 \lim _{t \rightarrow t^{\prime}}+w(x, t)=0$, so that the hypotheses of Theorem 8.4 are satisfied and $w(x, t) \equiv 0$ for $t^{\prime}<t<p-\delta$. Since $\delta$ is arbitrary, the theorem is proved. 
We now establish that a dual Poisson-Laguerre-Stieltjes transform belongs to $H^{*}$ in its region of absolute convergence.

THEOREM 10.2. Let

$$
u(x, t)=\int_{0}^{\infty} g(x, y ; t) d \alpha(y),
$$

the integral converging absolutely for $0<t<c$. Then $u(x, t) \in H^{*}$ there.

Proof. That $u(x, t) \in H$ for $0<t<c$ is a consequence of Theorem 8.2. To complete the proof, we must show that for all $t, t^{\prime}, 0<t^{\prime}<t<c$,

$$
u(x, t)=\int_{0}^{\infty} g\left(x, y ; t-t^{\prime}\right) u\left(y, t^{\prime}\right) d \Lambda(y) .
$$

Now, we have,

$$
\begin{aligned}
I & =\int_{0}^{\infty} g\left(x, y ; t-t^{\prime}\right) u\left(y, t^{\prime}\right) d \Lambda(y) \\
& =\int_{0}^{\infty} g\left(x, y ; t-t^{\prime}\right) d \Lambda(y) \int_{0}^{\infty} g\left(y, z ; t^{\prime}\right) d \alpha(z) \\
& =\int_{0}^{\infty} d \alpha(z) \int_{0}^{\infty} g\left(x, y ; t-t^{\prime}\right) g\left(y, z ; t^{\prime}\right) d \Lambda(y),
\end{aligned}
$$

with the change in order of integration valid by the assumed convergence of the integral defining $u\left(y, t^{\prime}\right)$. Hence, an appeal to Theorem 3.4, yields

$$
I=\int_{0}^{\infty} g(x, z ; t) d \alpha(z)
$$

which is $u(x, t)$ by hypothesis and the proof is complete.

The theorem may be strengthened by extending membership in $H^{*}$ in the region of conditional convergence of the dual Poisson-Laguerre-Stieltjes transform.

THEOREM 10.3. Let

$$
u(x, t)=\int_{0}^{\infty} g(x, y ; t) d \alpha(y)
$$

the integral converging for $0<t<c$. Then $u(x, t) \in H^{*}$ there.

Proof. By Corollary 7.4, we have, for $0<\delta<c / 2$,

$$
u(x, t)=O\left(\exp \left[x /\left(1-e^{-\delta / 2}\right)\right]\right), \quad x \rightarrow \infty,
$$

uniformly for $\delta<t \leqq c-\delta$, and by Theorem $8.2, u(x, t) \in H$ for $0<t<c$. Hence Theorem 10.1 establishes that

$$
u(x, t)=\int_{0}^{\infty} g\left(x, y ; t-t^{\prime}\right) u\left(y, t^{\prime}\right) d \Lambda(y), \quad 0<t^{\prime}<t<c,
$$


if the integral converges absolutely over that interval. That this is so is a consequence of Corollary 7.2 which for $0<t<t_{0}<c$ gives the estimate

$$
u(x, t)=O\left(\exp \left[x /\left(1-e^{t-t_{0}}\right)\right]\right), \quad x \rightarrow \infty,
$$

and therefore $u(x, t) \in H^{*}$.

We complete the section by proving that the integral of products of certain functions of $H^{*}$ is a constant.

THEOREM 10.4. Let $u(x, t) \in H^{*}$ for $a<t<b$ and $v(x, t) \in H^{*}$ for $a<-t<b$. If

$$
\int_{0}^{\infty}|u(y, t)| d \Lambda(y) \int_{0}^{\infty} g\left(y, z ; t^{\prime}-t\right)\left|v\left(z,-t^{\prime}\right)\right| d \Lambda(z)<\infty
$$

then

$$
\int_{0}^{\infty} u\left(x, t^{\prime}\right) v\left(x,-t^{\prime}\right) d \Lambda(x), \quad a<t<b
$$

is a constant.

Proof. By the definition of membership in $H^{*}$ we have, for $a<t^{\prime}<t<b$

$$
u(x, t)=\int_{0}^{\infty} g\left(x, y ; t-t^{\prime}\right) u\left(y, t^{\prime}\right) d \Lambda(y),
$$

and for $a<t<t^{n}<b$,

$$
v(x,-t)=\int_{0}^{\infty} g\left(x, z ; t^{\prime \prime}-t\right) v\left(z,-t^{\prime \prime}\right) d \Lambda(z)
$$

Then

$$
\begin{aligned}
I(t) & =\int_{0}^{\infty} u(x, t) v(x,-t) d \Lambda(x) \\
& =\int_{0}^{\infty} d \Lambda(x) \int_{0}^{\infty} g\left(x, y ; t-t^{\prime}\right) u\left(y, t^{\prime}\right) d \Lambda(y) \int_{0}^{\infty} g\left(x, z ; t^{\prime \prime}-t\right) v\left(z,-t^{\prime \prime}\right) d \Lambda(x) \\
& =\int_{0}^{\infty} u\left(y, t^{\prime}\right) d \Lambda(y) \int_{0}^{\infty} v\left(z,-t^{\prime \prime}\right) d \Lambda(z) \int_{0}^{\infty} g\left(x, y ; t-t^{\prime}\right) g\left(x, z ; t^{\prime \prime}-t\right) d \Lambda(x),
\end{aligned}
$$

where the interchange in order of integration is justified by (10.6). Now an appeal to Theorem 3.4 yields

$$
I(t)=\int_{0}^{\infty} u\left(y, t^{\prime}\right) d \Lambda(y) \int_{0}^{\infty} v\left(z,-t^{\prime \prime}\right) g\left(y, z ; t^{\prime \prime}-t^{\prime}\right) d \Lambda(z),
$$

and since $v \in H^{*}$, we have

$$
I(t)=\int_{0}^{\infty} u\left(y, t^{\prime}\right) v\left(y,-t^{\prime}\right) d \Lambda(y)=I\left(t^{\prime}\right)
$$

and the proof is complete.

11. Representation. Our goal now is to characterize those functions which are represented by dual Poisson-Laguerre-Stieltjes transforms of increasing functions. 
THEOREM 11.1. A necessary and sufficient condition that

$$
u(x, t)=\int_{0}^{\infty} g(x, y ; t) d \alpha(y), \quad 0 \leqq x<\infty,
$$

with $\alpha(y) \uparrow$ and the integral converging for $0<t<c$, is that $u(x, t) \geqq 0$ be in $H$ there.

Proof. The necessity of the condition is an immediate consequence of the fact that the kernel of the transform is a nonnegative member of $H$.

We establish the sufficiency of the condition by setting

$$
\beta_{\delta}(x)=\int_{0}^{x} g\left(y ; t_{0}\right) u(y, \delta) d \Lambda(y),
$$

$0<\delta<c, 0<t_{0}<c-\delta$. Then by Theorem 9.1 , we have

$$
0 \leqq \beta_{\delta}(x) \leqq \int_{0}^{\infty} g\left(y ; t_{0}\right) u(y, \delta) d \Lambda(y) \leqq u\left(0, t_{0}+\delta\right) .
$$

Moreover, by Corollary 9.3

$$
\begin{aligned}
u(x, t+\delta) & =\int_{0}^{\infty} g(x, y ; t) u(y, \delta) d \Lambda(y), \quad 0<t<c-\delta \\
& =\int_{0}^{\infty} \frac{g(x, y ; t)}{g\left(y ; t_{0}\right)} d \beta_{\delta}(y) .
\end{aligned}
$$

Hence

$$
u(x, t)=\lim _{\delta \rightarrow 0^{+}} \int_{0}^{\infty} \frac{g(x, y ; t)}{g\left(y, t_{0}\right)} d \beta_{\delta}(y), \quad 0<t<c
$$

By the Helly and Helly-Bray Theorems [14, pp. 26-52], it follows that

$$
u(x, t)=\int_{0}^{\infty} \frac{g(x, y ; t)}{g\left(y ; t_{0}\right)} d \beta(y)
$$

with $\beta(y) \uparrow$ and bounded. Thus

$$
u(x, t)=\int_{0}^{\infty} g(x, y ; t) d \alpha(y)
$$

where

$$
\alpha(y)=\int_{0}^{1} \frac{1}{g\left(u ; t_{0}\right)} d \beta(y) .
$$

It is clear that $\alpha(y) \uparrow$ and so the proof is complete.

We next derive criteria for the representation of functions by dual PoissonLaguerre-Stieltjes transforms of functions satisfying an integrability condition.

THEOREM 11.2. A necessary and sufficient condition that

$$
u(x, t)=\int_{0}^{\infty} g(x, y ; t) d \alpha(y), \quad 0<t<c
$$


with

$$
\int_{0}^{\infty} g(x ; c)|d \alpha(x)|<\infty
$$

is that $u(x, t) \in H$ for $0<t<c$ and

$$
\int_{0}^{\infty}|u(x, t)| g(x ; c-t) d \Lambda(x)<M, \quad 0<t<c .
$$

Proof. To establish the necessity of the condition, we note that by Theorem 5.1, (11.2) implies the convergence of the integral of (11.1) for $0<t<c$, so that by Theorem $8.2 u(x, t) \in H$ there. Further, by an appeal to Theorem 3.4, we have

$$
\begin{aligned}
\int_{0}^{\infty}|u(x, t)| g(x ; c-t) d \Lambda(x) & \leqq \int_{0}^{\infty} g(x ; c-t) d \Lambda(x) \int_{0}^{\infty} g(x, y ; t)|d \alpha(y)| \\
& =\int_{0}^{\infty}|d \alpha(y)| \int_{0}^{\infty} g(x ; c-t) g(x, y ; t) d \Lambda(x) \\
& =\int_{0}^{\infty} g(y ; c)|d \alpha(y)|<\infty
\end{aligned}
$$

where the change in order of integration is valid by Fubini's theorem. Hence the condition is necessary.

To prove the converse, set, for $h>0$,

$$
w_{h}(x, t)=\int_{0}^{\infty} g(x, y ; h) u(y, t) d \Lambda(y) .
$$

Then condition (11.4) implies, as a consequence of Theorem 5.1, that the integral defining $w_{h}(x, t)$ converges absolutely for $0<t<c-h$. Now, by (3.5),

$$
\left|w_{h}(x, t)\right| \leqq\left(\frac{e^{h}}{e^{h}-1}\right)^{\alpha+1} e^{x} \int_{0}^{\infty} \exp \left[-\left(\left(x e^{h}\right)^{1 / 2}-y^{1 / 2}\right) /\left(e^{h}-1\right)\right]|u(y, t)| d \Lambda(y),
$$

or, by (4.12) with $A=1 /\left(e^{c-t}-1\right)$, we have

$$
\begin{aligned}
&\left|w_{h}(x, t)\right| \leqq\left.\frac{e^{h}}{e^{h}-1}\right)^{\alpha+1} \exp \left[\frac{x}{\left(1-e^{h-c+t}\right)}\right] \\
& \times \int_{0}^{\infty} \exp \left[-\frac{y}{\left(e^{c-t}-1\right)}\right]|u(y, t)| d \Lambda(y) \\
&=\left[\frac{e^{h}\left(e^{c-t}-1\right)}{e^{c-t}\left(e^{h}-1\right)}\right]^{\alpha+1} \exp \left[\frac{x}{\left(1-e^{h-c+t}\right)}\right] \\
& \times \int_{0}^{\infty} g(y ; c-t)|u(y, t)| d \Lambda(y) .
\end{aligned}
$$

Hence, for $0<t<c-h-\varepsilon$, since (11.4) holds, we have

$$
\left|w_{h}(x, t)\right| \leqq K \exp \left[x /\left(1-e^{-\varepsilon}\right)\right] .
$$


An application of Theorem 10.1, since $w_{h}(x, t) \in H$, yields

$$
w_{h}(x, t)=\int_{0}^{\infty} g(x, y ; t-\delta) w_{h}(y, \delta) d \Lambda(y), \quad 0<\delta<t<c-h-\varepsilon,
$$

or

(11.9) $w_{h}(x, t+\delta)=\int_{0}^{\infty} g(x, y ; t) w_{h}(y, \delta) d \Lambda(y), \quad 0<\delta<t+\delta<c-h-\varepsilon$.

By (11.7), we have $w_{h}(y, \delta)=O\left(\exp \left[y /\left(1-e^{h-c+\delta}\right)\right]\right), y \rightarrow \infty$, and consequently, the integral of (11.9) converges absolutely for $0<\delta<t+\delta<c-h$. If we now let $h \rightarrow 0^{+}$, referring to (11.5) and Theorem 6.1, we have

$$
\lim _{h \rightarrow 0^{+}} w_{h}(x, t+\delta)=u(x ; t+\delta) .
$$

On the other hand, substituting the definition of $w_{h}(y, \delta)$ in (11.9), we have, on appealing to Theorem 3.4,

$$
\begin{aligned}
w_{h}(x, t+\delta) & =\int_{0}^{\infty} g(x, y ; t) d \Lambda(y) \int_{0}^{\infty} g(y, z ; h) u(z, \delta) d \Lambda(z) \\
& =\int_{0}^{\infty} u(z, \delta) d \Lambda(z) \int_{0}^{\infty} g(x, y ; t) g(y, z ; h) d \Lambda(y) \\
& =\int_{0}^{\infty} g(x, z ; t+h) u(z, \delta) d \Lambda(z)
\end{aligned}
$$

provided that the interchange in order of integration is justified. That this is so may be established by noting that

$$
\begin{aligned}
I= & \int_{0}^{\infty} g(x, z ; t+h)|u(z, \delta)| d \Lambda(z) \\
= & \int_{0}^{\infty} \frac{g(x, z ; t+h)}{g(z ; c-\delta)} g(z ; c-\delta)|u(z, \delta)| d \Lambda(z) \\
\leqq & K\left(\frac{e^{t+h-c+\delta}\left(e^{c-\delta}-1\right)}{e^{t+h}-1}\right)^{\alpha+1} \\
& \times \int_{0}^{\infty} \exp \left[\frac{-\left(\left(x e^{t+h}\right)-z^{1 / 2}\right)^{2}}{\left(e^{t+h}-1\right)}\right] \exp \left[\frac{z}{e^{c-\delta}-1}\right] g(z ; c-\delta)|u(z, \delta)| d \Lambda(z)
\end{aligned}
$$

or by (4.14) with $A=1 /\left(e^{c-\delta}-1\right)$, for $0<t+h<c-\delta$,

$$
\begin{aligned}
I \leqq & K\left(\frac{e^{t+h-c+\delta}\left(e^{c-\delta}-1\right)}{e^{t+h}-1}\right)^{\alpha+1} \exp \left[\frac{x e^{c-\delta}}{\left(e^{c-\delta}-e^{t+h}\right)}\right] \\
& \times \int_{0}^{\infty} g(z ; c-\delta)|u(z, \delta)| d \Lambda(z)
\end{aligned}
$$

which is finite by (11.4). Thus, (11.10) and (11.11) yield

$$
u(x, t+\delta)=\lim _{h \rightarrow 0^{+}} \int_{0}^{\infty} g(x, z ; t+h) u(z, \delta) d \Lambda(z)
$$


or since the integral is a continuous function of $h$,

$$
u(x, t+\delta)=\int_{0}^{\infty} g(x, z ; t) u(z, \delta) d \Lambda(z), \quad 0<\delta<t+\delta<c .
$$

Now, for some $c^{\prime}<c$, set

$$
\beta_{\delta}(x)=\int_{0}^{\infty} g\left(y ; c^{\prime}\right) u(y, \delta) d \Lambda(y)
$$

Then

$$
\begin{aligned}
\int_{0}^{\infty}\left|d \beta_{\delta}(x)\right| & =\int_{0}^{\infty} g\left(y ; c^{\prime}\right)|u(y, \delta)| d \Lambda(y) \\
& =\int_{0}^{\infty} \frac{g\left(y ; c^{\prime}\right)}{g(y ; c-\delta)} g(y ; c-\delta)|u(y, \delta)| d \Lambda(y) \\
& =\left[\frac{e^{c^{\prime}-c+\delta}\left(e^{c-\delta}-1\right)}{\left(e^{c^{\prime}}-1\right)}\right]^{\alpha+1} \int_{0}^{\infty} \frac{-\left(e^{c-\delta}-e^{c^{\prime}}\right) y}{\exp \left[\left(e^{c-\delta}-1\right)\left(e^{c^{\prime}}-1\right)\right]} \\
& \leqq\left[\frac{e^{c^{\prime}-c+\delta}\left(e^{c-\delta}-1\right)}{e^{c^{\prime}}-1}\right]^{\alpha+1} \int_{0}^{\infty} g(y ; c-\delta)(u(y, \delta)) d \Lambda(y)
\end{aligned}
$$

for $0<\delta<c-c^{\prime}$. But the integral converges by (11.4), and so

$$
\begin{aligned}
\int_{0}^{\infty}\left|d \beta_{\delta}(x)\right| & \leqq M\left(\frac{e^{c-\delta}-1}{e^{c^{\prime}}-1}\right)^{\alpha+1}, \quad 0<\delta<c-c^{\prime}, \\
& <M\left(\frac{e^{c}-1}{e^{c^{\prime}}-1}\right)^{\alpha+1} .
\end{aligned}
$$

The set of functions $\beta_{\delta}(x)$ thus has a total variation which is uniformly bounded. An application of the Helly theorem enables us to select a subset of $\beta_{\delta}(x)$ converging to a function $\beta(x)$ of bounded variation on $(0, \infty)$.

Letting $\delta \rightarrow 0$ through this subset, we have

$$
\begin{aligned}
\lim _{\delta \rightarrow 0} \int_{0}^{\infty} \frac{g(x, y ; t)}{g\left(y ; c^{\prime}\right)} d \beta_{\delta}(y) & =\lim _{\delta \rightarrow 0} \int_{0}^{\infty} g(x, y ; t) u(y, \delta) d \Lambda(y) \\
& =\lim _{\delta \rightarrow 0} u(x, t+\delta) .
\end{aligned}
$$

By the Helly-Bray theorem we obtain thus

$$
u(x, t)=\int_{0}^{\infty} \frac{g(x, y ; t)}{g\left(y ; c^{\prime}\right)} d \beta(y)=\int_{0}^{\infty} g(x, y ; t) d \alpha(y)
$$

where

$$
\alpha(y)=\int_{0}^{y} \frac{1}{g\left(u ; c^{\prime}\right)} d \beta(u)
$$


and the representation is established. Moreover,

$$
\int_{0}^{\infty} g(y ; c)|d \alpha(y)|=\int_{0}^{\infty}|d \beta(y)|<M\left(\frac{e^{c}-1}{e^{c^{\prime}}-1}\right)^{\alpha+1}
$$

by (11.15). Letting $c^{\prime} \rightarrow c$, we obtain (11.3) and the proof is complete.

\section{REFERENCES}

1. F. M. Cholewinski, Hankel convolution complex inversion theory, Mem. Amer. Math. Soc. No. 58 (1965), 67 pp.

2. F. M. Cholewinski and D. T. Haimo, The Weierstrass-Hankel convolution transform, J. Analyse Math. 17 (1966), 1-58.

3. - The Poisson-Laguerre transform, Bull. Amer. Math. Soc. 74 (1968), 137-139.

4. - The Weierstrass-Laguerre transform, J. Math. Anal. Appl. 23 (1968), 41-57.

5. A. Erdelyi et al., Higher transcendental functions, Vol. II, McGraw-Hill, New York, 1958,

6. - Tables of integral transforms, Vol. II, McGraw-Hill, New York, 1954.

7. D. T. Haimo, Integral equations associated with Hankel convolutions, Trans. Amer. Math, Soc. 116 (1965), 330-375.

8. — , The reduced Poisson-Hankel transform. I, Bul. Inst. Politehn. Iaşi 13 (1967), $39-42$.

9. - The reduced Poisson-Hankel transform. II, Bul. Inst. Politehn. Iaşi 14 (1968), 105-109.

10. I. I. Hirschman, Jr., Variation diminishing Hankel transforms, J. Analyse Math. 8 (1960-1961), 307-336.

11. - Laguerre transforms, Duke Math. J. 30 (1963), 495-510.

12. I. I. Hirschman, Jr. and D. V. Widder, The convolution transform, Princeton Univ. Press, Princeton, N. J., 1955.

13. S. Karlin and J. McGregor, Classical diffusion process and total positivity, J. Math. Anal. Appl. 1 (1960), 163-183.

14. D. V. Widder, The Laplace transform, Princeton Univ. Press, Princeton, N. J., 1941.

Clemson University,

Clemson, South Carolina

UNIVERSITY OF MisSOURI-ST. LOUIS,

ST. Louis, Missouri 